\title{
Biofilm Production by Organisms Causing Ventilator Associated Pneumonia
}

\author{
P. Nanthini Devi ${ }^{1^{*}}$ and S. Gomathi ${ }^{2}$ \\ ${ }^{1}$ Department of Microbiology, Sree Balaji Medical College and Hospital, Chennai, India \\ ${ }^{2}$ Department of Microbiology, SRM Medical College Hospital and Research centre, \\ Kattankulathur, India \\ *Corresponding author
}

\section{Keywords \\ Ventilator associated pneumonia, Biofilms, tissue culture plate method, Multidrug resistance}

\section{Article Info}

Accepted:

07 January 2018

Available Online:

10 February 2018

\section{A B S T R A C T}

Ventilator-associated pneumonia is defined as nosocomial pneumonia occurring more than 48 hours after patients have received mechanical ventilation. VAP in a critically ill patient significantly increases risk of mortality, ventilator time, length of stay and cost of care. The Endotracheal tube is a favourable site for biofilm formation. They are adherent cells which are embedded within a self-produced matrix of This EPS matrix impedes the normal functions of antibodies and the phagocytic cells of the immune system and leads to increased resistance to antibiotics. Aim of this study is to isolate and identify the organisms causing VAP and demonstrate biofilm production. A total of 149 patients were selected for the study. 40.94\% developed VAP. The organisms causing VAP was isolated and identified by conventional tests. The organisms were tested for biofilm production by the microtitre plate method using safranin dye and their optical densities measured. The organisms causing VAP were Acinetobacter baumannii, Pseudomonas aeruginosa, Escherichia coli, Klebsiella pneumoniae and Staphylococcus aureus. $47.73 \%$ were strong biofilm producers, $28.41 \%$ were moderate producers, $21.59 \%$ were weak producers and $2.27 \%$ did not produce any biofilm. Among the 42 isolated which produced strong biofilm, Acinetobacter baumannii was the most common organism associated with strong biofilm formation $(64.29 \%)$ followed by Klebsiella pneumoniae (21.43\%). The biofilm production by microorganisms is a threat because of its resistance to antimicrobial drugs. This highlights the importance of discovering new strategies focused in the removal of biofilm from the Endotracheal tube (ETT).Well planned and early extubations should be done to prevent biofilm formation on the ETT.

\section{Introduction}

Ventilator associated pneumonia (VAP) has been defined as pneumonia that occurs 48 hours after patients have received mechanical ventilation. Early onset VAP occurs within 4 days of mechanical ventilation and late onset
VAP occurs after 4 days (American Thoracic Society, 2005). It is the second most common nosocomial infection and the most common infection in ventilated patients. The prevalence varies between 9 to $27 \%$ with the maximum risk in the first five days after mechanical ventilation (Chastre and Fagon, 2002; 
Atul Ashok Kalanuria et al., 2014). VAP imposes increased length of stay in the intensive care unit and hospital and incurs extra financial burden to the patient (Eagye et al., 2009) (Fig. 2).

Biofilms are aggregates of microorganisms in which the cells are bound by a self-produced extracellular polymeric substance which adhere to a solid surface (Hall-Stoodley et al., 2004). The EPS components comprise extracellular DNA, proteins and polysaccharides (Lear and Lewis, 2012).

They can form on living or non-living surfaces and are due to cellular recognition sites on a surface, nutritional cues and exposure to sub inhibitory concentrations of antibiotics (Karatan and Watnick, 2009). Biofilms can include a single bacterial species or diverse and are protected from the body's immune system and antibiotics (Donlan, 2002).

Endotracheal tubes are used to provide ventilation to patients. They impair mucociliary clearance and facilitate accumulation of tracheo bronchial secretions (Craven and Steger, 1995). The presence of ET tube injures the mucosa and paves the way for the colonization of microorganisms and pneumonia.

It leads to the formation of biofilms which contribute to multi drug resistance (Rello et al., 1996). Staphylococcus aureus and Pseudomonas aeruginosa are the main organisms involved in biofilm formation although Acinetobacter, Streptococcus and Gram negative bacteria also can form bio films (Jamal et al., 2015). There are various methods to detect biofilm production like Tissue Culture Plate (TCP), Tube method (TM), Congo Red Agar method (CRA), bioluminescent assay, piezoelectric sensors and fluorescent microscopic examination (Hassan et al., 2011).

\section{Materials and Methods}

This was a prospective study done at the ICU complex of a tertiary care hospital in Chennai for a period of nine months.

\section{Inclusion criteria}

Patients who were admitted and underwent mechanical ventilation after two days (age > 18 years).

\section{Exclusion criteria}

Patients having Respiratory tract infection on admission like Pulmonary tuberculosis, COPD, Asthma and ARDS

A total of 149 patients on ventilator were included in the study. VAP was diagnosed on clinical and microbiological basis using the CPIS (Clinical Pulmonary Infection Score). The endotracheal aspirates were collected and processed with minimal delay. The organisms causing VAP were isolated and were subjected to Antimicrobial susceptibility testing including detection of various resistance mechanisms and screened for their ability to form biofilm by Tissue culture plate / Microtitre plate method.

\section{Microtitre plate method}

Colonies of the isolated bacteria causing VAP were grown on nutrient agar and inoculated in Trypticase soy broth (TSB) and incubated at $37^{\circ} \mathrm{C}$ for 24 hours. The culture was diluted 1:100 with freshly prepared trypticase soy broth.

A 96 well microtitre plate with flat bottom was taken and filled with $230 \mu \mathrm{l}$ of TSB. First three wells were filled with broth only to check for sterility and non- specific binding. Known positive and negative controls were inoculated in each plate. 
Then $20 \mu \mathrm{l}$ of the diluted cultures were added to the wells in triplicate and incubated at $37^{\circ}$ $\mathrm{C}$ for 24 hours. After incubation the plates were washed with phosphate buffer saline PBS ( $\mathrm{pH}-7.2$ ) to remove the free floating planktonic bacteria. $300 \mu \mathrm{l}$ of methanol was then added to each well and after 15 minutes, it was discarded and stained using $0.1 \%$ safranin dye.

After allowing standing for 20 minutes, the stain was discarded and washed with PBS. The adherent cells are resolubilised with $33 \%$ glacial acetic acid.

Optical densities (OD) of the stained adherent bacteria were measured with an ELISA auto reader at a wavelength of $490 \mathrm{~nm}$ (Fig. 1). The averages of the three OD values were taken. The OD readings were regarded as an index of bacteria attaching to the surface and forming biofilms. The cut off value of OD (ODc) was calculated (Mathur et al., 2006).

\section{Results and Discussion}

A total of 149 patients were included in this study out of which $101(67.78 \%)$ were males and $48(32.22 \%)$ were females. The incidence of VAP in our study was $40.94 \% \%$, with 61 of 149 patients developing VAP. The organisms isolated were Acinetobacter baumannii, Pseudomonas aeruginosa, Escherichia coli, Klebsiella pneumoniae and Staphylococcus aureus. Among the 61 VAP cases, $57.38 \%$ were monomicrobial (one bacterial species in tracheal aspirate) and $42.62 \%$ were polymicrobial (more than one bacteria in tracheal aspirate) yielding a total of 88 isolates.

$47.73 \%$ were strong biofilm producers, $28.41 \%$ were moderate producers, $21.59 \%$ were weak producers and $2.27 \%$ did not produce any biofilm. Among the 42 isolated which produced strong biofilms,
Acinetobacter baumannii was the most common organism associated with strong biofilm formation $(64.29 \%)$ followed by Klebsiella pneumoniae (21.43\%).

Among the moderate biofilm producers, $60 \%$ of Acinetobacter baumannii and $8 \%$ of Klebsiella pneumoniae isolates produced moderate biofilms. Among the weak biofilm producers, $36.84 \%$ of Acinetobacter baumannii and $31.58 \%$ of Klebsiella pneumoniae isolates produced weak biofilms.

\section{Correlation with multidrug resistance}

Multi drug resistance is defined as resistance to three anti-microbial classes. Among the VAP isolates, 69 isolates $(78.41 \%)$ were multi- drug resistant and 19 (21.59\%) were non multi -drug resistant. Acinetobacter baumannii produced $54.55 \%$ of multi drug resistance, Klebsiella pneumoniae $7.95 \%$, Pseudomonas aeruginosa $7.95 \%$, Staphylococcus aureus 5.68 and Escherichia coli $2.27 \%$.

Isolates with strong adherence in biofilm testing was found to be $36.63 \%$ multidrug resistant and isolates with moderate adherence in biofilm testing was found to be $21.59 \%$ multi drug resistant. Isolates with weak adherence in biofilm testing was found to be $19.32 \%$ multidrug resistant and non-adherent isolates were $1.14 \%$ multidrug resistant.

The use of various devices such as intravascular catheters, pacemakers, prosthetic joints has revolutionized the history of medical treatment. But the introduction of these artificial devices in the body has enabled the microorganisms to adhere and form complex biofilms which protect them from the body's immune system and the effect of antimicrobial drugs. Appropriate antibiotic therapy is ineffective in these cases and removal of the device becomes necessary. 
Fig.1 Showing microtitre plate with stained organisms

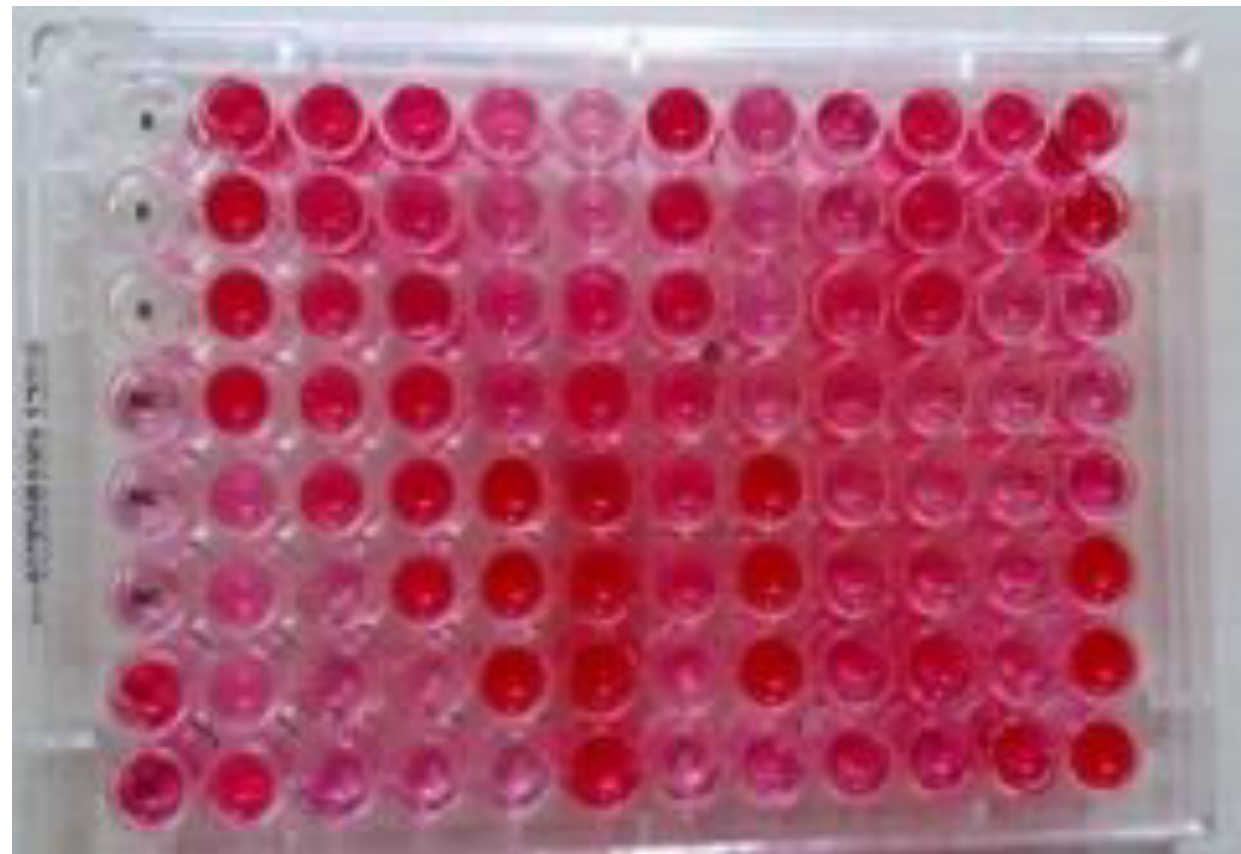

(B-Blank, PC-Positive Control, NC-Negative Control)

Fig.2 Microbiological profile of early and late onset VAP

\begin{tabular}{|c|c|c|c|c|c|}
\hline \multicolumn{6}{|l|}{$70.00 \%$} \\
\hline \multicolumn{6}{|l|}{$60.00 \%$} \\
\hline \multicolumn{6}{|l|}{$50.00 \%$} \\
\hline \multicolumn{6}{|l|}{$40.00 \%$} \\
\hline \multicolumn{6}{|l|}{$30.00 \%$} \\
\hline \multicolumn{6}{|l|}{$20.00 \%$} \\
\hline \multicolumn{6}{|l|}{$\begin{array}{r}10.00 \% \\
0.00 \%\end{array}$} \\
\hline D Late onset $V A_{P} 00 \%$ & & & & & \\
\hline Early onset VAP & MRSA & E.coli & $\begin{array}{c}\text { P.aerugin } \\
\text { osa }\end{array}$ & $\begin{array}{l}\text { K. pneum } \\
\text { oniae }\end{array}$ & $\begin{array}{c}\text { A.bauma } \\
\text { nnii }\end{array}$ \\
\hline Late onset VAP & $3.41 \%$ & $5.68 \%$ & $6.82 \%$ & $15.91 \%$ & $43.18 \%$ \\
\hline Early onset VAP & $2.27 \%$ & $2.27 \%$ & $2.27 \%$ & $3.41 \%$ & $14.77 \%$ \\
\hline
\end{tabular}


Fig.3 Adhesion profile of biofilm forming microorganisms causing VAP

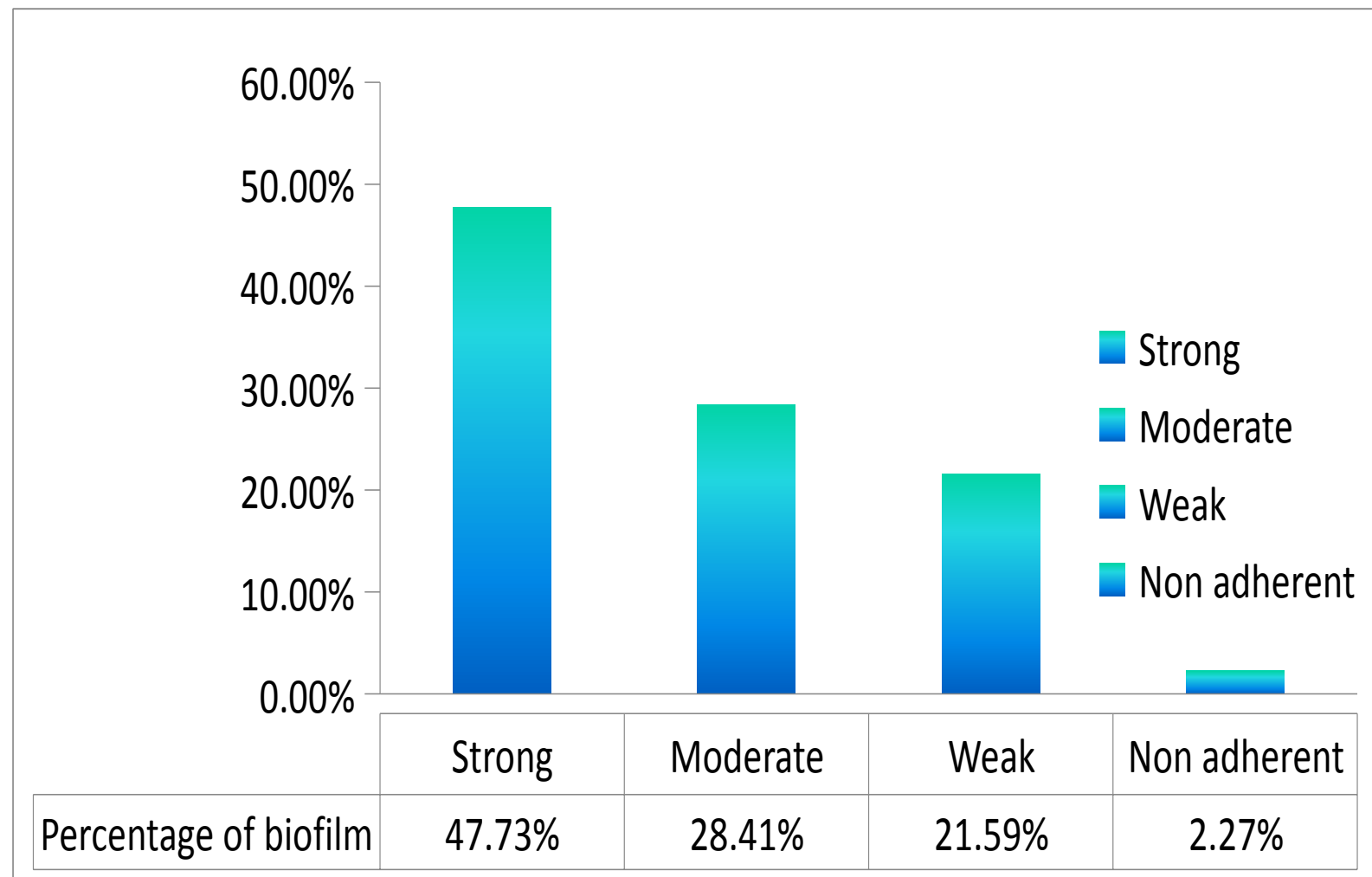

Table.1 Interpretation of biofilm production by microtitre plate method

\begin{tabular}{|l|l|l|}
\hline S. No & OD value & Biofilm \\
\hline 1. & OD $<$ ODc & Non-adherent \\
\hline 2. & ODc $<\mathrm{OD}<2$ ODc & Weak \\
\hline 3. & 2ODc $<$ OD $<4$ ODc & Moderate \\
\hline 4. & $>4$ ODc & Strong \\
\hline
\end{tabular}

Table.2 Results of biofilm estimation and their association with drug resistance

\begin{tabular}{|c|c|c|c|c|c|c|c|}
\hline \multirow[t]{2}{*}{ Organisms } & \multirow{2}{*}{$\begin{array}{l}\text { No of } \\
\text { organisms }\end{array}$} & \multicolumn{4}{|c|}{ Assessment of biofilms } & \multicolumn{2}{|c|}{ Drug resistance } \\
\hline & & Strong & Moderate & Weak & None & MDR & Non MDR \\
\hline $\begin{array}{l}\text { Acinetobacter } \\
\text { baumannii }\end{array}$ & 51 & 27 & 15 & 7 & 2 & 48 & 3 \\
\hline $\begin{array}{l}\text { Klebsiella } \\
\text { pneumoniae }\end{array}$ & 17 & 9 & 2 & 6 & - & 7 & 10 \\
\hline Escherichia coli & 8 & 3 & 2 & 3 & - & 2 & 6 \\
\hline $\begin{array}{l}\text { Pseudomonas } \\
\text { aeruginosa }\end{array}$ & 7 & 1 & 4 & 2 & - & 7 & - \\
\hline $\begin{array}{l}\text { Staphylococcus } \\
\text { aureus }\end{array}$ & 5 & 2 & 2 & 1 & - & 5 & - \\
\hline Total & 88 & $42(47.73 \%)$ & $25(28.41 \%)$ & $19(21.59 \%)$ & $2(2.27 \%)$ & $69(78.41 \%)$ & $19(21.59 \%)$ \\
\hline
\end{tabular}


Table.3 Correlation of biofilms adhesion with multidrug resistance

\begin{tabular}{|l|l|l|l|}
\hline S. NO & ADHIESION PROFILE & MIDR & NON MDR \\
\hline 1. & Strongly adherent & $32(36.36 \%)$ & $10(11.36 \%)$ \\
\hline 2. & Moderately adherent & $19(21.59 \%)$ & $6(6.82 \%)$ \\
\hline 3. & Weakly adherent & $17(19.32 \%)$ & $2(2.27 \%)$ \\
\hline 4. & Non adherent & $1(1.14 \%)$ & $1(1.14 \%)$ \\
\hline
\end{tabular}

The diagnosis of device associated infections and the role of biofilms should be considered crucial given its potential to cause life threatening nosocomial infections (Khardori and Yassien, 1995). There are various methods employed for the detection of biofilms such as Tissue Culture Plate, Tube method, Congo red agar method, bioluminescent assays and fluorescent microscopic examination (Hassan et al., 2011). Tissue culture plate method was found to be a better quantitative method when compared to other methods of biofilm detection (Hassan et al., 2011; Lima et al., 2017).

The total number of patients included was 149 out of which 101 (67.78\%) were males and 48 $(32.22 \%)$ were females. The incidence of VAP in our study was $40.94 \%$, with 61 of 149 patients developing VAP.

The organisms causing VAP were Acinetobacter baumannii, Pseudomonas aeruginosa, Escherichia coli, Klebsiella pneumoniae and Staphylococcus aureus. Among the 61 VAP cases, $57.38 \%$ were monomicrobial (one bacterial species in tracheal aspirate) and $42.62 \%$ were polymicrobial (more than one bacteria in tracheal aspirate) and the total number of isolates was 88 .

Acinetobacter had a propensity to produce $96.08 \%$ of biofilm production while the other pathogens Pseudomonas aeruginosa, Klebsiella pneumoniae, Escherichia coli and Staphylococcus aureus produced biofilms $100 \%$. Similar studies on biofilm detection revealed $50 \%$ and $62 \%$ of biofilm production among Acinetobacter baumannii isolates (Gurung et al., 2013; Rao et al., 2008). Another study showed $75 \%$ of biofilm production in Pseudomonas aeruginosa (Lima et al., 2017). Mathur et al., in their study found $53.9 \%$ biofilm production among Staphylococcus spp isolates (Mathur et al., 2006).

$47.73 \%$ were strong biofilm producers, $28.41 \%$ were moderate producers, $21.59 \%$ were weak producers and $2.27 \%$ did not produce any biofilm.

Among the 42 isolated which produced strong biofilms, Acinetobacter baumannii was the most common organism associated with strong biofilm formation $(64.29 \%)$ followed by Klebsiella pneumoniae (21.43\%). Shrinivasa Rao et al., described $62 \%$ of Acintobacter as a strong biofilm producer in their study (Rao et al., 2008).

Among the moderate biofilm producers, $60 \%$ of Acinetobacter baumannii and $8 \%$ of Klebsiella pneumoniae isolates produced moderate biofilms. Among the weak biofilm producers, $36.84 \%$ of Acinetobacter baumannii and $31.58 \%$ of Klebsiella pneumoniae isolates produced weak biofilms.

\section{Correlation with multidrug resistance}

Multi drug resistance is defined as resistance to three anti-microbial classes (Magiorakos et al., 2012). Among the VAP isolates, 69 isolates $(78.41 \%)$ were multi- drug resistant and $19(21.59 \%)$ were non multi -drug 
resistant. Acinetobacter baumannii produced $54.55 \%$ of multi drug resistance, Klebsiella pneumoniae $7.95 \%$, Pseudomonas aeruginosa $7.95 \%$, Staphylococcus aureus 5.68 and Escherichia coli $2.27 \%$. Similar study had a multidrug resistance of $66.1 \%$ with Acinetobacter as the predominant organism which was similar to our study (Mulla Summaiya and Jethwani Urmi, 2012).

Isolates with strong adherence in biofilm testing was found to be $36.63 \%$ multidrug resistant and isolates with moderate adherence in biofilm testing was found to be $21.59 \%$ multi drug resistant. Isolates with weak adherence in biofilm testing was found to be $19.32 \%$ multidrug resistant and non-adherent isolates were $1.14 \%$ multidrug resistant.

The presence of biofilms produced a higher rate of antibiotic resistance among bacteria. Acinetobacter had a tendency for strong biofilm production and multi drug resistance. There are no effective treatment strategies for biofilms in VAP patients.

The use of aerosolised antibiotics such as tobramycin, amikacin, colisthmethlate sodium has been found to have a reduction in respiratory infection signs and few studies have found no potential benefit (Palmer and Smaldone, 2014; Russell et al., 2016).

Silver coated endotracheal tube reduces adhesion of bacteria to devices in vitro and hinders with biofilm formation (Kollef et al., 2008; Berra et al., 2004) (Table 1-3 and Fig. 3).

There are many novel treatment options such as phage therapy, qurum sensing inhibition and induced biofilm dispersion. Further research should be done in this field to combat the problem of biofilm induced antibiotic resistance.

\section{References}

American Thoracic Society, Infectious Diseases Society of America: Guidelines for the management of adults with hospitalacquired, ventilator-associated, and healthcare-associated pneumonia. Am J Respir Crit Care Med 2005; 171: 388-416.

Atul Ashok Kalanuria, Wendy Zai, Marek Mirski. Ventilator-associated pneumonia in the ICU. Critical Care 2014; 18:208.

Berra L, De Marchi L, Yu ZX, Laquerriere P, Baccarelli A, Kolobow T. Endotracheal tubes coated with antiseptics decrease bacterial colonization of the ventilator circuits, lungs, and endotracheal tube. Anesthesiology. 2004 Jun; 100(6): 1446-56.

Chastre J, and Fagon JY: State of the art: ventilator-associated pneumonia. Am J Respir Crit Care Med 2002 165; 867-903.

Craven DE, and Steger KA. Chest. Epidemiology of nosocomial pneumonia. New perspectives on an old disease. 1995 Aug; 108(2 Suppl):1S-16S.

Donlan RM. Biofilms: Microbial Life on Surfaces. Emerging Infectious Diseases. 2002; 8(9):881-890.

Eagye KJ, Nicolau DP, Kuti JL. Impact of superinfection on hospital length of stay and costs in patients with ventilatorassociated pneumonia. Semin Respir Crit Care Med. 2009 Feb; 30(1):116-23.

Gurung J, Khyriem AB, Banik A, Lyngdoh WV, Choudhury B, Bhattacharyya P. Association of biofilm production with multidrug resistance among clinical isolates of Acinetobacter baumannii and Pseudomonas aeruginosa from intensive care unit. Indian Journal of Critical Care Medicine: Peer-reviewed, Official Publication of Indian Society of Critical Care Medicine. 2013; 17(4):214-218.

Hall-Stoodley L, Costerton JW, Stoodley P. Bacterial biofilms: from the natural environment to infectious diseases. Nature Reviews Microbiology. 2004; 2(2): 95-108.

Hassan A, Usman J, Kaleem F, Omair M, Khalid A, Iqbal M. Evaluation of different detection methods of biofilm formation in the clinical isolates. Braz J Infect Dis. 2011; 15(4): 305-11. 
Jamal M, Tasneem U, Hussain T and Andleeb S. Bacterial Biofilm: Its Composition, Formation and Role in Human Infections. RRJMB 2015; 4 (3):1-15.

Karatan E, and Watnick P. Signals, regulatory networks, and materials that build and break bacterial biofilms. Microbiology and Molecular Biology Reviews. 2009; 73 (2): 310-47.

Khardori N, and Yassien M. Biofilms in devicerelated infections. J Ind Microbiol. 1995 Sep; 15(3):141-7.

Kollef MH, Afessa B, Anzueto A, Veremakis C, Kerr KM, Margolis BD, et al., Silvercoated endotracheal tubes and incidence of ventilator-associated pneumonia: the NASCENT randomized trial. JAMA 2008; 300: 805-13.

Lear G, and Lewis GD, eds. 2012. Microbial Biofilms: Current Research and Applications. Caister Academic Press.

Lima JL da C, Alves LR, da Paz JNP, Rabelo MA, Maciel MAV, de Morais MMC. Analysis of biofilm production by clinical isolates of Pseudomonas aeruginosa from patients with ventilator-associated pneumonia. Revista Brasileira de Terapia Intensiva. 2017; 29(3):310-316.

Magiorakos AP, Srinivasan A, Carey RB et al., Multi drug-resistant, extensively drugresistant and pandrug-resistant bacteria: an international expert proposal for interim standard definitions for acquired resistance. Clinical Microbiology and Infection 2012; 18(3): 268-281.
Mathur T, Singhal S, Khan S, Upadhyay D J, Fatma T, Rattan A. Detection of biofilm formation among the clinical isolates of Staphylococci: An evaluation of three different screening methods. Indian $\mathbf{J}$ Med Microbiol 2006; 24:25-9.

Mulla Summaiya A, and N Jethwani Urmi. Assessment of biofilm formation by the causative organisms of Ventilator associated pneumonia at intensive care unit of a tertiary care hospital. National journal of medical research.2012; 2(1):15-18.

Palmer LB, and Smaldone GC. Reduction of bacterial resistance with inhaled antibiotics in the intensive care unit. Am J Respir Crit Care Med 2014; 189: 1225-33.

Rao RS, Karthika RU, Singh SP, Shashikala P, Kanungo R, Jayachandran S, Prashanth K. Correlation between biofilm production and multiple drug resistance in imipenem resistant clinical isolates of Acinetobacter baumannii. Indian J Med Microbiol. 2008 Oct-Dec; 26(4):333-7

Rello J, Soñora R, Jubert P, Artigas A, Rué M, Vallés J. Pneumonia in intubated patients: role of respiratory airway care. Am J Respir Crit Care Med. 1996 Jul; 154(1):111-5.

Russell CJ, Shiroishi MS, Siantz E, Wu BW, Patino CM. The use of inhaled antibiotic therapy in the treatment of ventilatorassociated pneumonia and tracheobronchitis: a systematic review. BMC Pulmonary Medicine. 2016; 16:40.

\section{How to cite this article:}

Nanthini Devi, P. and Gomathi, S. 2018. Biofilm Production by Organisms Causing Ventilator Associated Pneumonia. Int.J.Curr.Microbiol.App.Sci. 7(02): 486-493. doi: https://doi.org/10.20546/ijcmas.2018.702.061 